\title{
Direct Quotation in Japanese: A Site for Stereotyping
}

\author{
KATSUE AKIBA REYNOLDS \\ University of Hawai'i at Manoa
}

\section{Introduction}

Quotation of others' speech is one of the most common phenomena in the use of language. Discourses that we participate in as speakers/writers or hearers/readers, whether they are conversations, political speeches, conference papers, radio or TV news, novels, critiques, newspapers, magazines, or lectures, are full of quotations. Traditionally, quotations are identified either as "direct quotations" or as "indirect quotations", and it is generally assumed that a direct quotation is the exact reflection of what has actually been said. But how truthfully does a direct quotation reflect what has actually been said? Bakhtin (1981:340) observes that direct quotation is always subject to certain semantic changes no matter how accurately it is done. It is of great interest from a cognitive or sociolinguistic point of view to ask what the nature of the semantic changes is and how the changes occur. In this study I examine direct quotations in various Japanese texts, explore the mechanism of direct quotation, and demonstrate that direct quotation is the very site of stereotyping.

\section{From Primary Utterance to Quotation}

As preliminary observations, I first note the distinction between "primary speech genre" and "secondary speech genre" (Bakhtin 1986:62) and schematize the process of direct quotation as follows: Speaker A (primary speaker) says X (primary utterance), which expresses a set of meanings Xm (primary meanings); Speaker B (secondary speaker) interprets $\mathrm{Xm}$ as $\mathrm{Xm} 2$ and attempts to encode $\mathrm{Xm} 2$ into $\mathrm{X} 2$. In reality, there are cases in which the source of quotation is part of a non-conversational or written material, such as a public speech, a TV or newspaper news report, poetry, or a passage from a novel. In this exploratory study, however, I assume ordinary conversational interactions as the primary activity of language, and explore how semantic changes occur in the act of direct quotation. In other words, X is an utterance in "living conversation", while X2 may be part of a "living conversation" utterance or a written discourse.

Second, I wish to gain insight from the view that the signs of language, which are limited in number, must allow "built-in vagueness" in order to designate 
reality, which is infinite (Bolinger and Sears 1981:110). Stereotyping occurs in the area of non-propositional meanings or pragma-sociolinguistic discourse meanings, which are in most cases expressed without the speaker's conscious intention. Such meanings tend to be communicated by paralinguistic means (or "audible gestures" and "visible gestures" in Bolinger and Sears's terms), and are more analogic, vague, and variable than propositional meanings. The secondary speaker fails to fully recover the primary meanings partly because there is some built-in vagueness in the primary utterance and because the intended meanings cannot be exactly encoded back into the primary utterance. It is more than likely that the primary meanings are to be changed to greater degrees than generally considered. Japanese provides interesting examples to show how great the changes can be. I examine four kinds of Japanese discourse involving direct quotation, and I demonstrate how stereotypes can occur.

\section{Interactional Particles as "Audible Gestures"}

Japanese is known as a language with an extremely elaborate honorific system and a number of "sentence-final particles", among other things. (Since the term "sentence-final particle" is misleading, as I pointed out elsewhere, I refer to these particles as "interactional particles", and when I mention sentence-final particles I mean "interactional particles occurring in the sentence-final position.") Although honorifics and interactional particles intimately and animately interact in a total communicative act, they are different in linguistic status; honorifics are basically part of the system of Japanese grammar, the honorific and the non-honorific being morphosyntactically distinguished, while the particles are on the boundary between grammar and paralanguage. They convey more meanings by pronunciation than by the segmental form. They are "voice carriers." The intended meanings of particles cannot be clearly grasped without listening to the voice. Furthermore, particles carry different semantic values in different dialects (Reynolds 2000), which adds to the vagueness and complexity of the meanings to be conveyed by particles.

The verbal expressions $i k i$-masu (go-POLITE) and $i k u$ (go-Ø) both convey the propositional information '(I) will go', but they are lexically differentiated; the former is accompanied by the honorific suffix -masu and the latter is not. The verbal forms are either "polite" or "non-polite." The meanings to be conveyed by particles, on the other hand, are not categorically specifiable, but they convey different kinds of experience with different degrees of emphasis depending on the way they are pronounced. Particles can be pronounced with different lengths and different intonations signaling complex messages as to the speaker's attitudes and feelings towards the hearer or the content of the propositional information. Utterances such as ashita iku yo, ashita iku wa, ashita iku sa, ashita iku zo, and ashita $i k u z e$-in which the same proposition ashita iku ('tomorrow-go') is followed by a particle yo, wa, sa, zo, or ze-are all declarative statements meaning '(I) will go tomorrow'. The particles yo, wa, sa, zo, and ze added at the end do not affect the propositional meaning, but they are nevertheless an essential part of 
the communicative activity of the Japanese. Particles play major roles in stereotyping. In order to make my point regarding stereotyping in the process of direct quotation, I need to discuss "potential meanings" (Bolinger and Sears 1981:109) without examining this heretofore unexplored territory in any great detail. What I give in the following are therefore schematic descriptions of the major interactional particles utilized for speech stereotypes. Interrogatives (e.g. ka, kana, and kashira) and particle combinations (e.g. yo-na, yo-ne, wa-ne, wa-yo-ne) are not included here to keep our discussion from becoming unnecessarily convoluted. (cf. Reynolds 1985 for more details.)

Yo: Expresses the speaker's assertion with certainty and thus is used mainly by male speakers unless it combines with honorific verbs or other morphosyntactic adjustments as will be discussed below in connection with stylistic variations.

$\boldsymbol{W a}$ : The weakest in the assertion. This particle pronounced with a rising intonation is a clear indication of the feminine identity of the speaker. It is confusing, however, because wa pronounced with a kind of falling intonation is used even more frequently by males than female speakers in western dialects such as the Osaka dialect.

$\boldsymbol{S a}$ : Carries a tone of "strong insistence" and is said to be generally used by male speakers talking to their equals. However, it is rarely used as a sentencefinal particle in contemporary conversations, as shown immediately below.

Zo: Conveys the speaker's declarative and authoritative attitude and is used when the speaker has significantly greater power than the hearer does or when the speaker is doing a threatening act. It is therefore typically used by male speakers.

$Z \boldsymbol{e}$ : Similar to $z o$, but its meaning is more complicated than that of $z o$, perhaps because it is a fusion of $z o$ and $i / e$, an older particle conveying the speaker's intention to involve the hearer. This is also used exclusively by male speakers.

$\mathrm{Ne}$ : Expresses the speaker's intention to involve the hearer in judging the validity of the propositional part of the statement. The statement followed by this particle pronounced with a rising intonation becomes close to an interrogative.

$\mathbf{N a}$ : Similar to ne except that this is more imposing than ne, and is said therefore to be used by male speakers. In my data, however, it is used by females as well.

It is clear that particles play crucial roles in stereotyping, especially in regard to men's and women's speech. There are strong indications, however, that particles are "voice carriers" and the meanings that their audible gestures convey are rich but vague. Some of the subtle meanings to be conveyed by particles are inevitably changed or lost in the process of transmission from the primary speech to the secondary speech, spoken or written. 


\section{Interactional Particles in Conversation}

The first data concerning direct quotation is from the conversational data gathered by three Japanese sociologists for the purpose of their ethnomethodological study. ${ }^{1}$ I chose 21 sets of their transcriptions, romanized them, and made a database for my own research of conversational analysis including interactional particles and pause fillers. (I did not use the sets from which some pages were missing.) The table in (1) below is a summary of the counting of interactional particles found in three different positions: sentence-final position, sentenceinternal position, and sentence-external position (i.e. positions unrelated to the propositional structure of the sentence).

(1)

\section{Distribution of interactional particles}

\begin{tabular}{l||r|r|r|r|r|r|r||r} 
& $n e$ & $s a$ & $y o$ & $n a$ & $w a$ & $z o$ & $z e$ & TOTAL \\
\hline \hline Final & $\mathbf{1 4 7 0}$ & $\mathbf{0}$ & $\mathbf{4 4 5}$ & $\mathbf{1 1 6}$ & $\mathbf{9}$ & $\mathbf{3}$ & $\mathbf{2}$ & $\mathbf{1 8 1 7}$ \\
\hline Internal & 1737 & 647 & 2 & 0 & 0 & 0 & 0 & 2384 \\
\hline External & 74 & 0 & 0 & 0 & 0 & 0 & 0 & 74 \\
\hline TOTAL & 3281 & 647 & 447 & 116 & 9 & 3 & 2 & 4289
\end{tabular}

What is particularly relevant to this study of direct quotation is the fact that some particles occurred in great frequency, while others, especially those strongly gendered ones ( $w a$ for female speakers and $z o$ and $z e$ for males) were noticeably few. This limitation has recently been observed by Japanese researchers. Endo et al. (1989) found no instance of sentence-final $w a$ in their natural conversation data (2056 utterances), and Ozaki (1994) found only one instance in the 734 samples gathered from natural conversations. Furthermore, most of $w a, z o$, and ze in my data occurred in direct quotations when speakers were mimicking others. A female student of a co-ed college, for example, says the following using $w a$ in the final position of the quotation, imitating the feminine talk of women college students.

$$
\begin{aligned}
& \text { watashi kekkon-suru wa. } \\
& \text { I marry IP I } \\
& \text { 'I will get married.' }
\end{aligned}
$$

\footnotetext{
${ }^{1}$ Three sociologists, Ehara Yumiko, Yoshii Hiroaki, and Yamazaki Keiichi, recorded and transcribed 32 sets of 30-minute conversations (10 male-male pairs, 10 female-female pairs, and 12 male-female pairs). I am very grateful to these researchers for their generous permission for the use of their transcripts for my analysis. Their data, methodically gathered without losing the naturalness of the setting, gave me much confidence in my findings.

${ }^{2}$ Abbreviations in the word-by-word glossing: $\mathrm{COMP}=$ complementizer $(t o), \mathrm{COP}=$ copula $($ de'aru/da $), \mathrm{COP}=\mathrm{P}=$ polite/formal copula $($ desu $), \mathrm{DO}=$ direct object $(o), \mathrm{IP}=$ interactional particle, $\mathrm{NEG}=$ negative, $\mathrm{NOM}=$ nominalizer $(n o), \mathrm{SUBJ}=$ subject $(g a), \mathrm{TOP}=$ topic marker $(w a)$.
} 
Some comments on the result of the text count should be given at this point. First, the confirmative particle ne occurred more frequently in non-final positions than the final position. Second, $s a$, though it is described as a sentence-final particle in most studies of Japanese particles, did not occur at all as such in this data, while it occurred in internal positions with great frequency (647 instances). These are good reasons to consider it misleading to refer to all these particles as "sentence-final particles". Another point to be brought up is the fact that na, which occurred quite frequently, needs a closer examination. In the majority of cases, $n a$ was pronounced with a prolonged vowel, namely as naa (although the tapes were not available to me, the prolongation was clearly indicated in the Japanese transcriptions), and 37 instances of the total 106 occurred in female speakers' utterances. It is possible that particles that had come from different dialects are undergoing an overall change towards the formation of Standard Japanese, which began in the beginning of the modernization period of the history of Japan (i.e. sometime after the Meiji Restoration in 1868). I suspect that particles as used by dialect speakers or those which often appear as speech stereotypes in secondary genres, such as novels, popular songs, and TV dramas, may be mixed in the researchers' intuitive data.

At any rate, the data suggest a curious relationship between the use of sentence-final particles and stereotyping in direct quotations. To see the relationship more clearly, I examined direct quotations in three types of written conversational discourse: "magazine interviews", Japanese subtitles added to utterances of foreigners in TV news programs, and direct quotations in novels.

\section{Stereotyping in a Magazine Interview}

In Reynolds (1999), I compared the transcript of a TV interview of a Japanese woman (a primary genre text) and a "magazine interview" of the same woman (a secondary genre text). The woman, who was about 40 at the time, was confiding her sexual relationship with the then Prime Minister of Japan. First she talked with an editor of a popular weekly magazine and had him anonymously publish the story in the form of an interview, but she later appeared on TV to explain how it happened directly to the Japanese public. The difference between the TV interview and the magazine interview was striking.

The primary discourse is full of conversational features, such as incomplete sentences, pause fillers, afterthoughts, and instances of rephrasing and stammering. She talked decisively, assertively, hesitantly, or angrily depending upon the topic. She was very hesitant when she was asked to describe the scene in which the Prime Minister mentioned the price of her body, which she must have remembered as the most humiliating; she was determined and angry when she protested the comment that what had happened to her was just a question of the lower part of her body, not a political or social issue. It was evident on the TV screen that she was trying to appeal to the public with the utmost sincerity in spite of the difficulty that it was a question of human rights - a social issue. She was not performing the expected feminine role: there were no instances of the feminine 
particle $w a$ in her speech. In the magazine interview, on the other hand, an image of a woman with a different attitude was projected. Most of the 165 utterances in her responses were grammatically complete sentences ending with the polite finite form of the predicate, but with no hesitation. Of the 165 utterances, 80 were with a sentence-final particle. The table in (3) below is a summary of the 80 instances of particles.

(3) Particles used in the magazine interview

\begin{tabular}{c|c|c|c|c|c}
$y o$ & $n e$ & $w a$ & mono & other* & TOTAL \\
\hline 43 & 22 & 5 & 1 & 9 & 80 \\
Other" includes yone (combination of yo and $n e$ ) and $k a$ (interrogative)
\end{tabular}

Being dominated by the sense of finality of the complete sentences and assertion expressed by sentence-final particle yo, the magazine interview as a whole gave the impression that she was a woman who could talk about her own sexual matters without hesitation and in an imposing tone, in public. Many readers who were asked for comments after the publication of the magazine interview to be published in the subsequent issue of the magazine criticized her for being "nervy" and "shameless."

\section{Stereotyping Foreigners}

The second example to show that meaning changes in direct quotation is a set of brief interviews of foreigners telecast on Japanese news programs. It is a recent trend to include primary utterances of foreigners in the TV report with Japanese subtitles. The audience viewing interviews with foreigners on TV news programs hear the primary utterance in a foreign language and/or read the secondary utterance on the screen.

It has been noticed that the subtitles are often grossly inconsonant with the primary utterances in terms of the impression of the speaker. The gap suggests a problem inherent to the translation of non-propositional meanings developed in the socio-historical reality of each culture. The producers (or translators) face the difficult task of overcoming the semantic gap between the two languages/cultures. They first interpret the primary utterance into what they believe is the Japanese equivalent of the primary utterance, applying the knowledge about the audible and visible signs conventionalized in the Japanese semiotic system; then, by applying the Japanese rules of meaning-form correspondence, they encode the interpreted meanings into readily transcribable forms to show on the screen. In an attempt to read non-propositional meanings which may not exist or are not important in the semiotic system of the culture of the primary speaker, they must make more recourse not only to "audible" messages, but also to "visible" messages and must come up with translations that they think would make sense within the Japanese culture. In other words, the semantic changes from the primary utterance to the 
secondary utterance in this case involve not only stereotyping but also "Japanization."

I have analyzed 21 interviews telecast on FCI on January 1-2, 2001, in the two specials "Convergence: The Future TV News Programs Seen through the Eye of a TV Reporter" and "For Whom Are the Waves? Survival of Local Networks in the United States". Each piece was analyzed in terms of the speaker's gender, the professional status (affiliation and position), the attire (whether the clothes were formal, informal, or in between), and the language style as shown in the sentencefinal mode in the subtitle. Even though the interviewees were all media-related professionals and their speech was not particularly different from each other, their utterances were translated in four different discourse styles. The table in (4) below schematically illustrates each style with a verbal predicate meaning '(I) will write' consisting of a single verb in the middle column and a copular predicate meaning '(It) is Tokyo' made up of Tokyo and a copula in the right column. ${ }^{3}$

(4) Four stylistic variations used in Japanese translations

\begin{tabular}{|c|c|c|c|}
\hline \multicolumn{2}{|r|}{ Style } & '(I) write/will write' & '(It) is Tokyo' \\
\hline I & Neutral (written) & i. $\quad k a k u$ & v. Tokyo de'aru/da \\
\hline II & Formal (spoken) & ii. kaki-masu (yo) & vi. Tokyo desu (yo) \\
\hline III & Informal Male & iii. kaku $($ yo zo/ze...) & vii. Tokyo da (yo/zo/ze..) \\
\hline & Informal Female & iv. kaku wa/wa yo & viii. Tokyo yo/da wa yo \\
\hline
\end{tabular}

The table in (5) below is a summary of the analysis of the 21 interviews in the order they appeared in the two programs. (Judgment of the attire is based on the observations of three people, two Americans and myself. When the turn of the same interviewee consists of more than one sentence, the earlier ones are sometimes translated with the neutral style and the last one or two in one of the other styles. In such cases, the style in sentences at the end characterizes the whole turn. The spellings of names have been inferred from the Japanese katakana writing and may not be accurate.)

\footnotetext{
${ }^{3}$ Styles are not of course determined solely by the sentence-final modes, but they are indicated by many other features throughout the discourse. The choice of first-person pronouns such as watakushi, watashi, atashi, boku, and ore is particularly relevant to the stylistic diversity.
} 
Katsue Akiba Reynolds

(5) Interviewees on the FCI specials on January 1-2, 2000

\begin{tabular}{c|l|c|l|c|c} 
EX & NAME & M/F & POSITION & ATTIRE & STYLE \\
\hline \hline $1-1$ & Jason Austell & M & Reporter, KFMB & -formal & III \\
\hline $1-2$ & Bret Dumbron & M & Director, KFMB & +formal & II \\
\hline $1-3$ & --- & M & Technologist, WCYB & -formal & II \\
\hline $1-4$ & --- & M & Operator, WCYB & -formal & III \\
\hline $1-5$ & George Mazion & M & VP*, WCYB & +formal & II \\
\hline $1-6$ & Stacy Owen & F & Director, KRON & -formal & IV \\
\hline $1-7$ & Deborah McDermott & F & VP, YB* & +formal & II \\
\hline $1-8$ & Bob Goldbenger & M & Director, KNTB & -formal & III \\
\hline $1-9$ & Denis Walton & M & Representative, NAB* & +formal & II \\
\hline $2-1$ & Meril Brown & M & VP, MSNBC.com & -formal & II \\
\hline $2-2$ & Michael Oreskes & M & Mod., ABC News* & -formal & III \\
\hline $2-3$ & Nicholas Barretta & M & Pres., NEXTVENUE & +formal & III \\
\hline $2-4$ & Jane Applegate & F & Pres., sbtv.com & +formal & II \\
\hline $2-5$ & Teresa Ferguson & F & VP, DirectTV & +formal & I \\
\hline $2-6$ & Tim Butcher & M & VP, WebTV.net & -formal & II \\
\hline $2-7$ & Michael Pope & M & Prof., MIT & -formal & II \\
\hline $2-8$ & Alex Bentland & M & Prof., MIT & -formal & I \\
\hline 2-9 & Mitchel Lesnik & M & Prof., MIT & -formal & I \\
\hline $2-10$ & Richard Porpay & M & Prof., MIT & -formal & II \\
(YB: Young Broadcasting, Mod: moderator, NAB: National Association of Broadcasting)
\end{tabular}

In the TV interviews, Style I, being unmarked for non-propositional meanings, is used to provide only the content of the propositional meaning. Style II conveys a sense of formality similar to the $\mathrm{V}$-form in the theory of power semantics (Brown and Gilman 1960). It can be followed by interactional particles when used in face-to-face interactions. At the informal level the gender difference becomes apparent: in the male style, the non-polite verb can be followed by any declarative particle; in the female style, on the other hand, it can be followed by only wa or $w a-y o$, and various verbs need to be modified towards weaker assertion. The informal copula $d a$ of the copula nominal or adjectival predicate is typically suppressed in the female style as seen from a comparison between the male style Tokyo da yo and the female style Tokyo yo in the table in (4). Related to this nonuse of the assertive copula $d a$ is the pattern [S no], which is commonly appropriated in the speech of female speakers. Although the no at the end of this construction is sometimes identified as a feminine sentence-final particle, from a formal point of view, it is a nominalizer. The $[\mathrm{S} n o]$ is the zero-copula version of the $[\mathrm{S}$ no desu/da] construction and is roughly translated into English with 'it is that $\mathrm{S}$ ' (Kuno 1973:223-233). Compare (6) and (7). 
Direct Quotation in Japanese

(6) unzari shite-i-ta no. ..... wakuwaku suru wa. disgusted be-PAST NOM excited be IP '(It is that) I have been disgusted. . . (I'm) excited.' isogashiku naru yo. san-ji kara bangumi ga hajimaru $n$ da.
busy $\quad$ become IP 3-o'clock from program SUBJ begin NOM COP
'(We will) become busy. It is that the program will begin at three o'clock.'

Example (6) consisting of [S $n o]$ and [S $w a]$ is from the speech of Stacy Owen, a female news director of KRON, and (7) with [S yo] and [S $n d a]$ (no being contracted) is from the speech of a male staff member of WCYB. Since Japanese counterparts of these professionals would never talk in these informal styles in similar contexts, it is curious why and how the Japanese translators make such stylistic distinctions. Audible and visible gestures as well as social variables, such as gender, age, and social status, seem to be important clues.

If the speaker is a senior male with a distinguished title, wearing a formal coat and tie, the subtitle would be in Style II (e.g. 1-2, 1-5). If the speaker is a male speaker in casual work clothes, it will be in Style III (e.g. 1-1, 2-2). A female media person is interpreted as a user of the formal spoken style if she is wearing, for example, a jacket with a necklace on (e.g. 1-7, 2-4), but if she is relatively young and wearing casual clothes, she is likely to be represented by the informal women's style as in (6), in which all the assertive force of the utterance is reduced or eliminated. There are of course cases in which perceived semiotic indicators are contradictory or confusing to the interpreter. For example, sample 2-3 is a male company president wearing a coat and tie, but his utterances were translated by a mix of Style I and Style III, suggesting that the translator somehow sensed a relatively strong maleness in him although he was by other standards a person who would talk in Style II. It may be his fast speech with three instances of quite noticeable stammering (they will $\mid$ they $\mid$ they are..., in $\mid$ in terms of..., it $\mid$ it' it's...), three quick pauses and four instances of the pause filler ah within the 46second turn, or sharp penetrating eyes and boldness in the front-namely, "visible gestures" - that made the translator perceive him as an aggressive masculine man. Sample 2-9 was an MIT professor wearing a sweatshirt instead of a formal coat and tie, which is expected of a prestigious university professor in Japan. His utterances were translated in Style I, the unmarked style.

The examples in these programs are not particularly special. I have observed these news programs for years, transcribing many instances showing significant semantic changes from the primary to the secondary. A farmer in South America, men on the street in Israel after the presidential election, and male earthquake victims in El Salvador were all represented in the informal male speech style. The distressed and angry shouts of a Russian woman and mother of a crew member who died in a submarine accident, protesting to a Russian official, were translated with informal women's style. The anger expressed not only in her voice but also in her whole body and the Japanese female language style was an extremely 
Katsue Akiba Reynolds

awkward mismatch. The female language style is for Japanese women, who are not supposed to ever show anger.

The stereotyping of foreigners' discourse according to Japanese ideology is a serious problem emerging as Japanese TV programs become increasingly internationalized. During the Atlanta Olympics, a university teacher wrote a letter to the editor of the magazine Asahi, pointing out that Canadian gold medalist Donovan Bailey, who "spoke in a voice with confidence and with humility" in what the college teacher perceived as proper English, "even sounding like a thoughtful philosopher," was stereotyped as an "unsophisticated, coarse male" when his interview was translated with the informal male Japanese style. The author of the letter wrote, "The translation is awfully unpleasant to me: the translator is too insensitive...Isn't this a form of racism?" Yabe (1996) examined all the direct quotations (marked by quotation marks) that appeared in the three major dailies during the Atlanta Olympics: all the Japanese athletes, men and women, responded with the formal polite style (Style II), while foreigners' utterances were translated with different styles, female athletes' comments with the informal female speech style and the comments by male athletes, particularly non-whites, were translated with the informal masculine style.

\section{Creating Novel Characters}

The third place where one can see the gap between primary and secondary genre speech is the novel. Bakhtin defines the novel as an artistically organized diversity of social speech types and individual voices, and states:

\footnotetext{
The internal stratification of any single national language into social dialects, characteristic group behavior, professional jargons, genetic languages, languages of generations and age groups, tendentious languages, languages of authorities, of various circles and of passing fashions, languages that serve the specific sociopolitical purposes of the day, even of the hour...this internal stratification present in every language at any given moment of its historical existence is the indispensable prerequisite for the novel as a genre. (Bakhtin 1981:262-3)
}

The diversity is most concretely observed in the characters' dialogues, which are direct quotations in the sense that they represent the voices of others in a fictitious world. Characters' dialogues can be far more extreme in stereotyping because of the inevitable loss of voice in the process of going from spoken to written discourse and because of the stylization chosen by the author, abstracting some relevant characteristics of social and individual speech types, to be maximally effective for the artistic purposes. To support this point, I have analyzed four major characters of Hard-Boiled Wonderland and The End of the World (Wonderland, hereafter), a novel of nightmare fantasy by Murakami Haruki (1949-). ${ }^{4}$

\footnotetext{
${ }^{4}$ Murakami is known in the West through English translations, such as The Wind-Up Bird Chronicle and Norwegian Wood.
} 
The characters of this twilight zone are deprived of their shadows and do not have any memory of their past. None of the characters are given personal names. They are referred to by second-person pronouns (e.g. anata, anta, omae, and kimi) in the dialogues and by their categories (e.g. "Old Man," "Gatekeeper," "Fatty Woman in Pink Dress," and "Librarian Girl") or by the gendered third-person pronouns kare (male) and kanojo (female) in the narratives. More interesting is the final mode where detailed interactional meanings are created through interactions of various linguistic dimensions, such as formal vs. non-formal verbals, classical vs. contemporary lexical items, and the presence vs. non-presence of interactional particles. I have examined the first 100 utterances of each of the four characters talking to the author-narrator and have conducted a text count analysis of their final modes.

The "old man" character, who hired the narrator for his research purpose, is a biologist with some connection with "the central system" of the Wonderland. His utterances are consistently in Style II, suggesting his seniority and status. The method of achieving this stylistic level is not by the standard formal verb suffix, but by the [S desu] construction, a somewhat deviant variety of the [S no desu] construction. He says kaku desu ('I will write') instead of kaki-masu or kaku no desu. 39 of the 100 samples are in this peculiar construction, 7 in the standard [S no desu] construction, and 3 in the [S wake desu] construction (wake being a variant of the nominalizer). There are only 3 instances of verbals with -masu. What is more peculiar in the speech of the biologist is the particle na following the desu of the [S desu] construction and the desu of the regular nominal or adjectival predicate, as seen in (8) below.

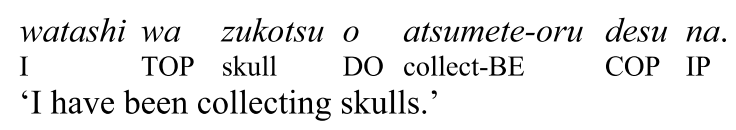

All 14 instances of na follow desu, thus making the utterance-final mode desu na a unique feature of this character's speech. The interactional particle $z o$, the one with the greatest degree of assertion, combined with desu is also found in the speech of the same character, though not as frequently as the mode desu na.

Final modes, such as desu na and desu zo, may sound unusual and contradictory since desu conveys formality, politeness, distance, etc., and the particles $n a$ and zo carry the opposite semantic values, such as masculine intimacy or intention to involve the hearer, but they actually occur in the speech of some male speakers. The appropriation of this mode as a feature of the biologist's speech is not totally unrealistic. It is the frequency of this mode in his speech that is unrealistic and responsible for stereotyping.

The speech of the gatekeeper to the Wonderland is almost the opposite of the biologist's. He is depicted as a virile man of lower status. He talks exclusively in Style III, constantly using the informal copula $d a$ or masculine particles sa and $n a$ as shown in (9)-(11) below. 
(9) anta mo kawatta hito da na. you also different person COP IP

'You are also a strange person.'

(10) furui yume o yomи $n \quad d a$. old dreams DO read NOM COP '(You) will read old dreams.'

(11) wakaru sa.

understand IP

'(I) understand (you).'

What $[S d e s u]$ is to the biologist is what $[\mathrm{S} n d a]$ is to the gatekeeper. 20 of the 100 samples of the gatekeeper's speech are in this form. Counting the utterances ending with the $d a$ of the regular copular predicate, 55 end with $d a$, with the particle of strong assertion yo (3 cases), the particle of masculine intimacy na (12) or $s a(9)$, or with no particle. The speech of the gatekeeper, totally lacking the formality required in talk with a stranger, gives the impression that he is "uncivilized."

The speech of the female characters is not very different from person to person. Both the librarian and the biologist's granddaughter talk in the informal female style (Style IV). 21 of the first 100 utterances of the librarian's speech end with wa. Also salient is the frequent use of [S no], the zero-copula version of the [S no $d a$ ] construction. 42 of the 100 samples are in this form. In addition, the copula of the regular copula sentence is always left out as in (12) below.

$\begin{array}{llll}\text { (12) koko wa totemo shizukana machi yo. } & \text { miet } & \text { town IP }\end{array}$

'This is a very quiet town.'

The biologist's granddaughter's speech is the same as the female librarian's although she is much younger.

There are some other linguistic devices with which the author attempts to achieve his intended image of Wonderland. What I have shown, however, must be sufficient to see how only certain meanings are disproportionately expanded and how grossly characters are stereotyped.

\section{Conclusions}

In this study, primary genre data and several sets of secondary genre data were comparatively analyzed in terms of the semantic changes in the process of direct quotation. The data amply demonstrated that semantic changes in the transmission of another's speech are inevitable, and it is indeed true that "we stereotype" (Lakoff 1990:181). We are constantly exposed to speech stereotypes when we converse with others, read magazines, watch $\mathrm{TV}$, and read novels, with little 
awareness of what is happening, resulting in unconscious collaboration with the stereotyping. Japanese linguists, including myself, have attempted to describe the pragma-sociolinguistic meanings of interactional particles and other ingredients of stereotyping, taking primary conversational data and secondary data indiscriminately all together. Consequently we have not only been prevented from an adequate understanding of those areas of the language, but we have also helped further enforce existing stereotypes. I wish to emphasize here that as the college teacher wrote to Asahi, stereotyping can be harmful and it functions as a conservative force preserving old ideology. The media needs to be more sensitive to the socio-psychological ramifications of their language use instead of continuing to indulge in existing stereotypes. Even novels should not be completely exempt from the accusation of stereotyping merely on the grounds that it is necessary for artistic creation. Several novels by Murakami Haruki including Wonderland have been translated into English such that most of the meanings conveyed by speech stereotypes in the Japanese original have been lost, but the value of the novels is nevertheless appreciated. The book reviewer of The Atlantic, for example, has commented that Wonderland is a provocative work "rich in action, suspense, odd characters and unexpected trifles." Also, there are younger Japanese writers who are creative in reflecting in characters' speech the emerging linguistic diversity in contemporary Japanese. Language abounds in resources to create styles better suited to equality and globalization. Linguistics can suggest ways that stereotypes are produced and can show the harmful consequences of categorization.

\section{References}

Bakhtin, M. M. 1981. The Dialogic Imagination. Austin: University of Texas Press.

Bakhtin, M. M. 1986. The Problems of Speech Genres. In Caryl Emerson and Michael Holquist (ed.), Speech Genres and Other Late Essays (trans. Vern W. McGee). Austin: University of Texas Press, 60-102.

Bolinger, D. L., and D. A. Sears. 1981. Aspects of Language. New York: Harcourt Brace Jovanovich.

Brown, R., and A. Gilman. 1960. The Pronouns of Power and Solidarity. In T. A. Sebeok (ed.), Style in Language. New York: John Wiley, 253-276.

Chafe, Wallace L. 1982. Integration and Involvement in Speaking, Writing and Oral Literature. In Deborah Tannen (ed.), Spoken and Written Language: Exploring Orality and Literacy. Norwood, NJ: ABLEX, 35-53.

Ehara, Yumiko, Yoshii Hiro'aki, and Yamazaki Keiichi. 1993. Seisabetsu no Esunomesodorogii [An Ethnomethodological Study of Sex Discrimination]. In Reynolds (ed.), Onna to Kotoba [Women and Language]. Tokyo: Yushindo, 189-228.

Endo, Orie, et al. 1989. Josei no Hanashi Kotoba-Terebi no Intavyuu Bangumi kara [Women's Spoken Japanese-From Interviews on TV]. Kotoba 17. 
Katsue Akiba Reynolds

Kuno, Susumu. 1973. The Structure of the Japanese Language. Cambridge, MA: MIT Press.

Lakoff, Robin. 1990. Talking Power. New York: Harper Collins.

Ozaki Yoshimitsu. 1994. Shuu Joshi ni-tsuite: Datsu-Josei-Sen'yoo-Go no Keikoo [On Sentence-Final Particles: Tendency towards De-genderization]. A Report of the Tokyo Josei Zaidan Grant Project.

Reynolds, Katsue Akiba. 1985. Female Speakers of Japanese. Feminist Issues 5(2):13-46.

Reynolds, Katsue Akiba. 1999. Discourse and Social Change. Kotoba 20:18-34.

Reynolds, Katsue Akiba. 2000. A Sociohistorical Perspective: Implications of a Quantitative Analysis of Japanese Interactional Particles. Kotoba 21:88-103.

Yabe, Hiroko. 1996. Shinbun Hodo no Gaikokujin Danwa ni Miru Dan-jo-sa [The Gender Difference in Foreigners' Speech Observed in Newspaper Reports]. Kotoba 17:58-72.

University of Hawai'i at Manoa

Department of East Asian Languages and Literatures

1890 East-West Road, Moore 361

Honolulu, HI 96822

katsue@hawaii.edu 